\title{
Francine Descarries
}

Sociologue, professeure-chercheure, département de sociologie, UQAM

(1998)

\section{"Le projet féministe à l'aube du XXIe siècle: un projet de libération et de solidarité qui fait toujours sens"}

Un document produit en version numérique par Jean-Marie Tremblay, bénévole, professeur de sociologie au Cégep de Chicoutimi

Courriel: jmt_sociologue@videotron.ca

Site web pédagogique : http://www.uqac.ca/jmt-sociologue/

Dans le cadre de la collection: "Les classiques des sciences sociales"

Site web: http://www.uqac.ca/Classiques_des_sciences_sociales/

Une collection développée en collaboration avec la Bibliothèque

Paul-Émile-Boulet de l'Université du Québec à Chicoutimi

Site web: http://bibliotheque.uqac.uquebec.ca/index.htm 
Cette édition électronique a été réalisée par Jean-Marie Tremblay, bénévole, professeur de sociologie au Cégep de Chicoutimi à partir de :

\section{Francine Descarries,}

"Le projet féministe à l'aube du XXIe siècle: un projet de libération et de solidarité qui fait toujours sens". Un article publié dans la revue Cahiers de recherche sociologique, no 30, 1998, pp. 179-210. Montréal : Département de sociologie, UQAM.

Mme Francine Descarries est directrice universitaire, Alliance de recherche IREF/Relais-Femmes, et professeure au Département de sociologie de l'Université du Québec à Montréal (UQAM)

[Autorisation formelle accordée par Mme Descarries de diffuser ce texte accordée le 18 juillet 2004]

Courriel : descarries.francine@uqam.ca

Polices de caractères utilisée :

Pour le texte: Times, 12 points.

Pour les citations : Times 10 points.

Pour les notes de bas de page : Times, 10 points.

Édition électronique réalisée avec le traitement de textes Microsoft Word 2001 pour Macintosh.

Mise en page sur papier format

LETTRE (US letter), 8.5' x 11',)

Édition numérique réalisée le 8 mai 2005 à Chicoutimi, Ville de Saguenay, province de Québec, Canada.

\section{Fait avec}

Macintosh 


\title{
Table des matières
}

\author{
Introduction
}

I. Trois courants, trois approches de la division sociale des sexes et des stratégies de libération
a) Le féminisme égalitaire
b) La réponse des radicales
c) La réconciliation du féminin et du maternel

II. La fin des grands courants: vers un féminisme solidaire Vers un féminisme pluriel, pluraliste et solidaire

Résumé 


\section{Francine Descarries,}

directrice universitaire, Alliance de recherche IREF/Relais-Femmes, et professeure au Département de sociologie de l'UQAM

\section{"Le projet féministe à l'aube du XXIe siècle: un projet de libération et de solidarité qui fait toujours sens".}

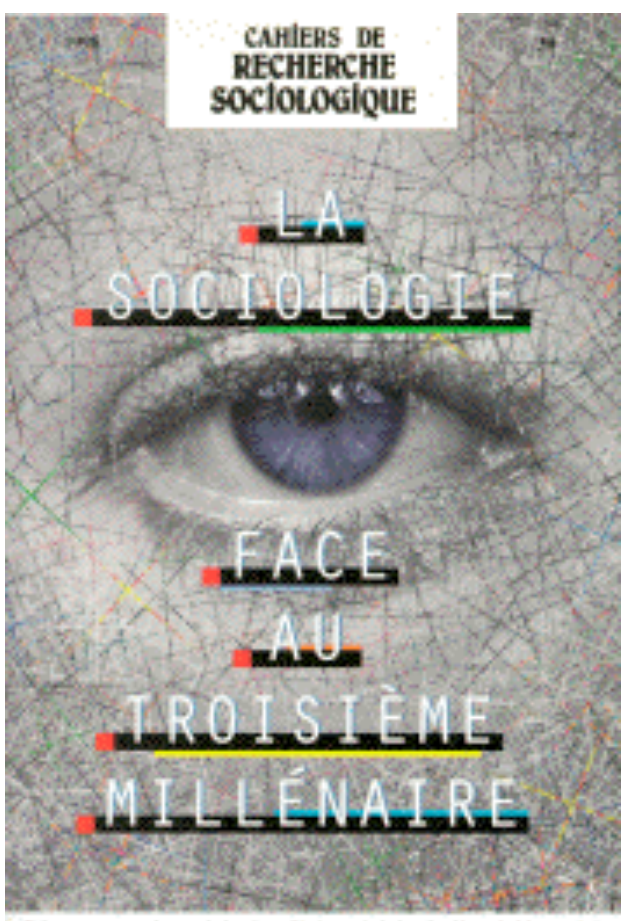

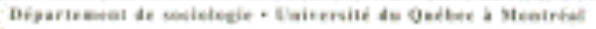

Un article publié dans la revue Cahiers de recherche sociologique, no 30, 1998, pp. 179-210. Montréal : Département de sociologie, UQAM. 
Francine Descarries,

directrice universitaire, Alliance de recherche IREF/Relais-Femmes, professeure au Département de sociologie de l'UQAM

"Le projet féministe à l'aube du XXIe siècle:

un projet de libération et de solidarité qui fait toujours sens". "

\section{Introduction}

\section{Retour à la table des matières}

Les dernières décennies ont été le théâtre de la re-naissance du mouvement féministe dans plusieurs régions du monde. Celui-ci s'est déployé à la fois comme maître d'œuvre d'un ralliement démocratique sans précédent autour d'une contestation de l'omniprésence des catégories de sexe dans l'organisation et la structuration des sociétés et comme discours critique des conditions de production et de reproduction des rapports sociaux de sexe. Il est difficile de saisir l'ampleur de l'événement historique que constituent l'émergence contemporaine d'un savoir et d'une pratique féministes, puis leur intégration, tout aussi imparfaite soit-elle, dans les champs de la connaissance et des représentations sociales.

Je suis redevable, pour la préparation de ce texte, à Céline Séguin qui a réalisé une analyse préliminaire de la littérature, Féminisme et post-modernisme: vers une alternative féministe post-moderne? Je la remercie de m'avoir permis d'y puiser inspiration et propositions analytiques. Je tiens également à remercier ma collègue et collaboratrice de longue date Christine Corbeil pour nos stimulantes discussions, ainsi que pour son attentive relecture du présent article et ses commentaires pertinents. 
Certes, l'énonciation par les femmes d'une parole «autre», tout comme l'expression de revendications concernant l'égalité, est loin d'être un phénomène contemporain. En font foi les traces anciennes et éloquentes laissées par les Christine de Pisan (1364-1430), Aphra Behn (1640-1680), Olympe de Gouges (1745-1793), Mary Wollstonecraft (1759-1797), Flora Tristan (1803-1844), Elizabeth Cady Stanton (1815-1902) et Susan B. Anthony (1820-1906), pour ne citer que quelques-uns des noms qui ont réussi à passer à la postérité ${ }^{1}$. Pourtant, ce n'est qu'au cours du présent siècle, et dans un nombre limité de sociétés, que tombent progressivement les barrières et les sanctions institutionnelles et structurelles mises en place par des ordres sociaux profondément androcentristes. Et ce n'est encore que plus récemment que des femmes, sans être dans une position d'exception 2, ont réussi à imposer comme légitime et digne de durer leur participation au monde des idées et de la science ${ }^{3}$.

Né de la révolte des femmes à l'égard des normes et des conditions qui président à leur destin personnel et à leur confinement dans la sphère privée, le mouvement féministe contemporain est rapidement devenu, au cours des années soixante-dix, un projet intellectuel et politique pour penser et agir le Nous femmes, annoncé par la parution du Deuxième sexe de Simone de Beauvoir en 1949 et rendu possible à imaginer à la suite des premières grandes mobilisations collectives des années soixante. Pratiques de résistance, les visées de changement du mouvement des

La première, Christine de Pisan (La cité des Dames [1404-1405], Paris, Stock, 1986), dénonce les oublis volontaires de l'histoire au sujet du rôle joué par les femmes dans l'édification des sociétés et la façon dont ses contemporains traitent les femmes. Auteure prolifique, aventurière, abolitionniste et féministe avant les termes, Aphra Behn placera la contestation du pouvoir masculin au cœur de son oeuvre et de son action politique, dès la présentation de sa première oeuvre théâtrale: The Force Marriage (1670). En septembre 1791, en réponse à la Déclaration des droits de l'homme et du citoyen, Olympe de Gouges rédige sa propre Déclaration des droits de la femme et de la citoyenne. En 1792, une éducatrice britannique, Mary Wollstonecraft, publie à son tour un essai sur La défense des droits de la femme (Paris, Petite Bibliothèque Payot, 1973; titre original anglais: A Vindication of the Rights of Woman). En collaboration avec Lucretia Mott (1793-1880), Elizabeth Cady Stanton a été l'instigatrice du premier congrès féministe, Women's Rights Convention, qui s'est tenu à Seneca Falls, aux États-Unis, en 1848. EIle est également, avec Susan B. Anthony et d'autres activistes de l'époque, co-auteure d'une série de volumes sur la lutte pour le suffrage féminin aux États-Unis

2 Sous ce rapport, Éliane Gubin rappelle que, pour souligner l'attribution d'un deuxième prix Nobel à Marie Curie, le journal Le Matin, dans sa livraison du 7 novembre 1911, écrivait au sujet de cette dernière: «Nous ne lui attribuons de passion que pour la science, d'attachement qu'au travail. Son oeuvre nous fait oublier son sexe.» (Éliane Gubin, «Créer. Hier et aujourd'hui», Sextant. Revue du Groupe interdisciplinaire d'études sur les femmes, Bruxelles, ULB, été 1994, p. 6.)

3 Mes propos sont inspirés par l'œuvre de Dale Spender, Women of Ideas and what Men Have Done to them from Aphra Behn to Adrienne Rich, Londres, Routledge and Kegan Paul, 1982. Voir également, de la même auteure, Feminist Theorists: Three Centuries of Women's Intellectual Tradition, Londres, The Women's Press, 1983. Je remercie la direction des Cahiers de recherche sociologique qui, respectueuse d'une pratique féministe qui vise à rendre plus visibles les femmes et leurs écrits, a accepté de déroger à ses conventions éditoriales et à donner au long le prénom des auteures dans les références bibliographiques. 
femmes ont rapidement mené militantes et intellectuelles à vouloir appréhender autrement la dynamique sexuée des rapports sociaux, à démystifier une tradition intellectuelle et scientifique qui était parvenue jusque-là à les exclure de ses lieux de production, et à poser, suivant en cela la proposition de Ti-Grace Atkinson, le travail de théorisation comme acte militant ${ }^{4}$. Dans cette optique, les études féministes poursuivront une démarche intellectuelle concertée, bien que par ailleurs éclatée, pour venir à bout des présupposés androcentristes des savoirs dominants, échapper à la rigidité de leurs propositions normatives, se soustraire à l'enfermement de la pensée binaire et statique du féminin et du masculin et mettre en évidence, là où ils étaient invisibles ou occultés, les processus sexués à l'œuvre dans la structuration du social et des connaissances. Plusieurs propositions seront formulées et diverses approches seront conçues en vue de contrer les effets pervers d'une organisation sociale dans laquelle les places et les activités des individus sont naturalisées et hiérarchisées en fonction de leur appartenance de sexe.

Résultat de «négociations complexe ${ }^{5}$ », mouvantes et continues, entre, d'une part, l'impérieuse nécessité théorique d'interpeller, du point de vue des femmes, un savoir dominant construit a peu près exclusivement à partir de l'expérience et des représentations des hommes et, d'autre part, la non moins impérieuse nécessité d'engager, d'alimenter des luttes politiques et le militantisme au quotidien, les études féministes s'énoncent, depuis leur émergence, à travers de multiples voix, de multiples lieux de production et évoluent en diverses directions. Elles sont tour à tour critiques épistémologiques des biais sexistes du savoir et de sa prétendue neutralité; réfutations des modèles théoriques dominants proposés pour penser et dire les femmes et leur vie; interrogations sur la condition des femmes et leur position dans l'histoire; écritures littéraires pour échapper à l'enfermement et à l'exclusion du langage androcentriste; réflexions politiques engagées en faveur d'un idéal de démocratie et de transformation des institutions sociales qui ont légitimé et reconduit, au fil du temps, la construction sociale et culturelle des sexes. Les études féministes sont également, et plus spécifiquement, démarches sociologiques pour constituer les femmes en catégorie sociale et poser le sexe/genre comme catégorie critique d'analyse, de même que méthodologies pour déconstruire les représentations et les mécanismes reconstitutifs de la division sociale des sexes et des autres systèmes de domination.

Ti-Grace Atkinson, «Le nationalisme féminin», Nouvelles questions féministes, nos 6-7, 1984, p. 35-54.

5 Elizabeth Grosz, $<<$ Sexual difference and the problem of essentialism $>>$, dans Naomi Schor et Elizabeth Weed (dir.), The Essential Difference, Bloomington, Indiana University Press, 1994, p. 82-97. 
Partie intégrante d'un mouvement social majeur et de ses enjeux, les études féministes ${ }^{6}$ contribuent ainsi au renouvellement des savoirs, à l'instauration de l'incertitude face à la prétendue objectivité des sciences instituées, à la transformation des pratiques et à la formulation d'une vision autre de la société. Dans cette perspective, elles ont, du moins en Occident, fait accepter l'idée qu'il n'était plus possible de penser et d'agir le monde comme avant, c'est-à-dire d'une façon qui «derrière la catégorie abstraite de l'humain universel a systématiquement gommé, exclu ou refoulé les expériences de la moitié, voire de la majorité du genre humain ${ }^{7}{ }_{\gg}$. De même, elles ont forcé l'élargissement de l'espace de créativité et d'observation des disciplines et amené ces dernières, avec plus ou moins de succès, à s'engager dans un exercice d'inventivité pour concevoir «les transformations que la présence des femmes peut apporter dans le champ culturel général ${ }^{8} \gg$ et dans tous les autres aspects de la vie sociale.

En inscrivant les rapports sociaux de sexe au cœur de leur élaboration conceptuelle et en faisant de la transformation de ces derniers un enjeu stratégique, les études féministes se sont donc progressivement imposées dans l'univers des sciences de l'Homme comme démarche critique et problématique du changement. Dans un continuum dont la complexité mais aussi la richesse découlent de la diversité et de l'interrelation des perspectives adoptées ainsi que des oppositions observées entre celles-ci, elles ont énoncé, au cours de leur évolution, plusieurs propositions théoriques pour interpeller la division et la hiérarchie inhérentes à la sexuation des rapports sociaux dans tous les champs du social et produire des sujets-femmes autonomes, actrices de leur propre vie et du jeu politique dans un procès égalitaire de libération. Ce faisant, elles ambitionnent de soustraire les femmes, en tant que catégorie sociale, «de l'emprise du même et de la généralité de la féminité sous laquelle le patriarcat les a enfouies ${ }^{9} \gg$ en démontrant que, d'une part, les rapports de sexe font système ${ }^{10}$, et que, d'autre part, dans ce système, «la catégorisation de sexe

6 Dans le contexte des sciences humaines et sociales, on désigne sous le terme études féministes un champ pluridisciplinaire de connaissances qui s'est développé en milieu universitaire depuis la fin des années soixante-dix. Ni domaine d'études uniquement centrées sur les femmes ni courant homogène de pensée, les études féministes se penchent sur différentes problématiques et font appel à divers outils conceptuels et à diverses méthodologies pour analyser la dimension sexuée des rapports sociaux de hiérarchisation et de division à l'œuvre dans nos sociétés, de même que les représentations sociales et les pratiques qui les accompagnent, les modèlent et les remodèlent.

7 Eleni Varikas, «Féminisme, modernité, post-modernisme: pour un dialogue des deux côtés de l'océan», dans Denis Berger, Féminismes au présent, Paris, L'Harmattan, 1993, p. 59.

8 Marcelle Marini, «D'une création minoritaire à une création universelle», Les Cahiers du GRIF, Paris, Éditions Tierce, no 45, automne 1990, p. 53.

9 Françoise Collin, «Le féminisme et la crise du moderne», dans Diane Lamoureux, Fragments et collages: essai sue le féminisme québécois des années '70, Montréal, Éditions du remue-ménage, 1986, p. 12.

10 Cette thèse est au cœur de l'œuvre de Christine Delphy depuis la parution de «L'ennemi principal», Partisans, vol. 54-55, 1970, p. 157-172. 
est un processus dynamique par lequel la position de chacun des groupes de sexe par rapport à l'autre est sans cesse redéfinie ${ }^{11}{ }$, recomposée, ainsi que nommée et distinguée, selon les époques et les lieux.

La pensée féministe actuelle, ou, devrait-on dire, les pensées féministes offrent-elles encore ces mêmes possibilités, participent-elles à ces mêmes finalités? Sont-elles en mesure d'imaginer un nouveau contrat entre les sexes? Peuvent-elles être porteuses «d'autres définitions du mouvement [des femmes] et de son sujet politique ${ }^{12} \gg$ ? Enfin, sont-elles capables d'alimenter une lutte féministe solidaire, une politique de coalition ouverte aux diversités et aux différences, «sans tomber dans le piège d'une fragmentation à l'infini ${ }^{13} \gg$ ? Voilà quelques-unes des interrogations qui constituent le fil conducteur du présent essai. Dans cette optique, j'argumenterai que l'avenir des études féministes - ou d'une sociologie des rapports de sexe - dépendra de leur capacité à analyser les problèmes et les contradictions qui découlent tant des choix que des refus formulés par les femmes au cours des dernières décennies que de l'insistance qu'elles mettent à préserver, tout en la redéfinissant, l'identité féminine. L'apport normatif et stratégique des études féministes, ajouterais-je, est pour sa part lié à leur efficacité a préserver la conscience d'un destin commun de femmes qui traverse l'ensemble des rapports sociaux, tout en étant attentives aux diversités introduites non seulement par l'hétérogénéité et la diversité des identités, des préoccupations et des expériences de femmes, tant à l'échelle locale qu'à l'échelle nationale et planétaire, mais encore par les «déplacements frontaliers de l'inégalité et les incessantes décompositions et recompositions des partages ${ }^{14} \gg$ et des hiérarchies entre les sexes.

Indéniablement, les mises en garde contre les généralisations abusives ou les prétentions à une fausse universalité, réitérées à satiété au cours de la dernière décennie par les féministes américaines post-structuralistes - nous y reviendrons -, ne peuvent être balayées du revers de la main. Elles sont en soi fructueuses, ne serait-ce que parce qu'elles signalent l'effritement du potentiel explicatif et mobilisateur des grands récits qui ambitionnaient de tout expliquer. Elles incitent, par voie de conséquence, au développement de réflexions théoriques mieux situées et moins inféodées aux perspectives dominantes, de même qu'à l'élaboration de stratégies de

11 Anne-Marie Daune-Richard et Anne-Marie Devreux, «Rapports sociaux de sexe et conceptualisation sociologique», Recherches féministes, vol. 5, no 2, 1992, p. 26.

12 Colette St-Hilaire, «Le féminisme et la nostalgie des grands Récits», Cahiers de recherche sociologique, no 23, 1994, p. 80.

13 Laura L. Frader, «Histoire des féminismes, différences et identités de classe en France au XXe siècle», dans Ephesia, La place des femmes. Les enjeux de l'identité et de l'égalité au regard des sciences sociales, actes de la IVe Conférence mondiale sur les femmes, Paris, La Découverte, 1995 , p. 359.

14 Michelle Perrot, «Identité, égalité, différence. Le regard de l'histoire», dans Ephesia, ouvrage cité, p. 46. 
solidarité, plutôt que consensuelles, au sein du mouvement des femmes. Au cours de la dernière décennie, ce type d'admonitions a largement refaçonné le visage des études féministes en orientant la teneur de leurs débats vers les problématiques de l'identité, de l'égalité et de la différence des sexes ${ }^{15}$.

Pour ma part, l'analyse des écrits des théoriciennes dont les noms sont le plus fréquemment associés à la critique féministe post-moderniste m'amène à postuler que ni le seul recours à une alternative féministe anti-rationnelle ni la seule évocation des canons de la modernité ne permettront de répondre adéquatement aux exigences théoriques et aux enjeux politiques auxquels fait face aujourd'hui le mouvement des femmes ${ }^{16}$. Mais avant de pousser plus loin cette réflexion, il y a lieu d'effectuer un bref retour sur le trois principaux courants de pensée qui sont, selon Moi ${ }^{17}$, à l'origine du foisonnement des idées, du choix des thèmes et de la teneur des débats au sein des études féministes. D'autant que ces trois courants, qui constituent le corpus théorique sur lequel se sont construites les études féministes, sont actuellement revisités par les avancées et polémiques qui traversent les études féministes, particulièrement en ce qui concerne les concepts de rapports de pouvoir, de genre, d'identité féminine, de différence/différences ou d'universalité. Le premier courant est le féminisme égalitaire ou universaliste qui est à l'origine de la résurgence du mouvement au cours des années soixante et de l'adoption d'une cause des femmes; à ce jour, il continue d'informer l'action d'un grand nombre d'organisations féministes et d'organismes gouvernementaux et syndicaux. Le second courant est le féminisme radical. Celui-ci occupe une large partie de l'espace théorique durant les années soixante-dix et propose une lecture féministe des rapports sociaux de sexe en termes de dominant et dominée. Certaines tendances de ce courant se redéploieront sous diverses formes, au cours des années quatre-vingt-dix. Elles peuvent être regroupées autour d'un mouvement souvent désigné sous le vocable de féminisme global ${ }^{18}$, mais que, pour

15 Ces trois notions ont fait l'objet d'un important colloque de recherche à Paris, en 1995, dans le cadre de la préparation de là participation française à la Ive Conférence des Nations unies sur les femmes tenue à Beijing. Les actes de ce colloque, La place des femmes. Les enjeux de l'identité et de l'égalité au regard des sciences sociales, ont déjà été cités dans des notes précédentes.

16 Parmi les ouvrages qui offrent une intéressante lecture des débats en cours entre post-modernisme, post-structuralisme et féminisme, mentionnons: Seyla Benhabib, Judith Butler, Drucilla Cornell et Nancy Franser, Feminist Contentions. A Philosophical Exchange, New York, Routledge, 1995; Naomi Schor et Elizabeth Weed, ouvrage cité; Linda Alcoff et Elizabeth Potter, Feminist Epistemologies, New York, Routledge, 1993; Linda Nicholson (dir.), Feminism/Postmodernism, New York, Routledge, 1990; Chris Weedon, Feminist Practice and Poststructuralist Theory, New York, Basil Blackwell, 1987.

17 J'ai déjà proposé cette classification dans un essai antérieur réalisé en collaboration avec Shirley Roy, Le mouvement des femmes et ses courants de pensée: essai de typologie, Ottawa, Cahiers de l'Icref, 1988.

18 Désigné également sous le vocable Integrative Feminisms par Angela Miles dans son ouvrage Integrative Feminisms. Building Global Visions, 1960-1990s (New York, Routledge, 1996). Sur les perspectives du féminisme global, on consultera avec intérêt: Charlotte Bunch, «Organizing for 
ma part, je préfère appeler féminisme solidaire ${ }^{19}$. Enfin, le troisième courant est celui du féminisme de la fémelléité ${ }^{20}$, qui se développe parallèlement à différentes tendances des féminismes égalitaire et radical. Il s'imposera au cours de la décennie 1980 comme vision alternative pour revaloriser l'expérience concrète ou symbolique des femmes et donner aux dimensions privées de leur vie (enfantement, soins et éducation des enfants, relations intimes) une expression éthique et esthétique. Il faut ici insister sur l'idée qu'une telle catégorisation, ainsi que les appellations employées pour désigner les courants de pensée relevés en tant que dominants, demeure une proposition ouverte et non exclusive de classification. Elle n'est utilisée que pour permettre de mieux se repérer dans l'effervescence et la mouvance des idées et des propositions théoriques présentées par les études féministes depuis plus de trente ans. Elle ne doit surtout pas servir a enfermer la parole des femmes dans une gaine rigide: les frontières entre chacun des courants ne sont absolument pas étanches et sont appelées à se déplacer selon les auteures, les lieux de production et les périodes. De même, faut-il le rappeler, la prédominance d'un courant de pensée au cours d'un moment historique donné ne signifie nullement la disparition des autres propositions qui l'ont précédé dans le temps.

women's human rights globally», dans Joanna Kerr, Ours by Right: Women's Rights as Human Rights, Londres, Zed Press, 1993, p. 141-194; Maria Mies, «The global is the local», dans Rosiska Darcy de Oliviera et Thais Corral (dir.), Terra Femina, Brazil, mai-juin 1992, p. 54-68; Chandra Talpade Mohanty, Ann Russo et Lourdes Torres (dir.), Third World Women and the Politics of Feminism, Bloomington, Indiana University Press, 199 1; Jessie Bernard, The Female World from a Global Perspective, Bloomington, Indiana University Press, 1987.

19 Voir Francine Descarries et Christine Corbeil, «Égalité, autonomie et survie. Du rêve à la réalité: les pratiques du mouvement des femmes», Nouvelles Pratiques sociales, automne 1997, p. 19-28.

20 En proposant ce néologisme, Shirley Roy et moi-même voulions mettre l'accent sur le fait que ce courant de pensée désignait comme lieu du féminin un espace charnière qui se situerait entre le biologique - l'expérience du corps - et le psychologique et qui en constituerait la synthèse. 


\section{I. \\ Trois courants, trois approches de la division sociale des sexes et des stratégies de libération}

$\underline{\text { Retour à la table des matières }}$

Au-delà de leur passion pour l'avènement d'une société non sexiste et de leur convergence au cœur d'un système d'énonces, qui visent à fournir un nouveau sens aux rapports sociaux de sexe et à faire émerger des sujets femmes pleinement autonomes, les théories du féminisme peuvent être départagées notamment selon les distinctions qu'elles font dans leur lecture de la division sociale des sexes et de la problématique de la libération. Les propositions qu'elles avancent ne sont nullement réductibles les unes aux autres. Elles se distinguent tant par leur filiation théorique, leurs outils conceptuels et leur vision du monde que par le lieu ou la nature de leur regard, les dimensions d'analyse qu'elles privilégient et les enjeux relationnels et socio-politiques qu'elles font ou non surgir. C'est pourquoi, dès sa résurgence au cours des années soixante, le mouvement féministe contemporain ne sera pas le lieu d'une seule parole, d'un unique programme ou d'un dessein homogène. A cette époque, tout comme aujourd'hui, ses problématiques logent à plusieurs enseignes. Elles portent la trace du contexte socioculturel de leur émergence, tout en étant profondément marquées par les cultures intellectuelles et organisationnelles dominantes des grands centres où elles ont d'abord été énoncées et agies: Etats-Unis, France et Grande-Bretagne. 


\section{a) Le féminisme égalitaire}

\section{$\underline{\text { Retour à la table des matières }}$}

Le féminisme égalitaire représente sans nul doute la problématique qui a réussi à rejoindre le plus grand nombre de femmes depuis l'appel lancé par Betty Friedan en $1963{ }^{21}$. Héritier de la tradition des suffragettes et des mouvements antiracistes américains, il revendique l'égalité de droit et de fait pour toutes les femmes au nom du droit inaliénable de chaque individu à l'égalité et à l'autodétermination. Principalement «tourné vers l'action ${ }^{22}$ », il se préoccupe peu de produire une analyse critique des sources de l'inégalité. L'égalité des sexes y est posée comme finalité sans exégèse et son acceptation découle d'une adhésion à la pensée libérale. Pour les féministes égalitaires, les rôles socialement imposés dans la division sexuelle du travail constituent la principale source de discrimination et de conflits entre les sexes, alors que les injustices subies par les femmes sont interprétées comme des relents de conditions socioculturelles anachroniques ${ }^{23}$. L'accès à l'égalité passe prioritairement par l'abolition des conditions discriminatoires vécues par les femmes dans les sphères de l'éducation, du travail et de la politique. Dans cette perspective, le mot d'ordre est de modifier la socialisation et l'éducation des filles, de réaménager les tâches domestiques au sein de la famille et de favoriser l'accès des femmes aux lieux de savoir et de pouvoir économique ou politique. Il s'agit, en somme, d'œuvrer à la transformation des mentalités et des pratiques familiales et publiques - et non pas nécessairement des rôles - afin que femmes et hommes puissent réaliser leur potentiel en tant qu'individus libres et autonomes. Idéologiquement fondé sur un parti pris optimiste par rapport à la capacité de réforme du système patriarcal, le courant égalitaire mise sur la volonté et l'investissement des femmes pour gagner une égalité de chances avec les hommes. Il constitue en quelque sorte la plate-forme modérée du mouvement des femmes.

21 Betty Friedan, La femme mystifiée, Paris, Gonthier, 1964 (version anglaise, 1963).

22 Cette expression est de Ginette Castro, Radioscopie du féminisme américain, Paris, Presses de la Fondation nationale des sciences politiques, 1984.

23 Jessie Bernard, dans Born Female: The High Cost of Keeping Women Down (New York, McKay, 1968), est l'une des premières à avoir problématisé les coûts de la discrimination sexuelle. Du côté de la France, il faut consulter Andrée Michel, Activité professionnelle de la femme et vie conjugale, Paris, Éditions du CNRS, 1974. Le recueil d'essais dirigé par Vivian Gornick et Barbara K. Moran, Women in Sexist Society (New York, Signet Book, 1971), présente un éventail représentatif des thèmes abordés à l'époque. Marie-Agnès Barrère-Maurisson et autres, dans Le sexe du travail (Grenoble, Presses universitaires de Grenoble, 1984), offrent un panorama intéressant des travaux réalisés en France dans cette optique. Parmi les travaux plus récents, soulignons l'excellent ouvrage de Geneviève Fraisse, La différence des sexes, Paris, PUF, 1995. 


\section{b) La réponse des radicales}

\section{$\underline{\text { Retour à la table des matières }}$}

À partir des années soixante-dix, les problématiques radicales vont dominer la mouvance intellectuelle et politique du mouvement des femmes ${ }^{24}$. Reprenant certains thèmes annonces par Simone de Beauvoir (1949), tout en se distanciant des quelques «scories naturalistes et sexistes que sa pensée charrie ${ }^{25}$ », elles prennent forme dans le refus des féministes de se définir à travers «la relation de tutelle qui les liait au socialisme ${ }^{26} \gg$. Mais si les féministes radicales s'inscrivent en faux contre le précepte le socialisme d'abord et le reste suivra, elles n'acceptent pas non plus de s'associer à la revendication des égalitaristes: des emplois d'abord et tout le reste suivra. Car, là où ces dernières demeurent attachées aux normes familiales, aux règles du jeu politique et à l'individualisme de la société libérale, les féministes radicales s'opposent a toute solution de compromis ${ }^{27}$. Là où les féministes égalitaires ne voient que les anachronismes et les ratés d'un système perfectible, les féministes radicales voient un ordre patriarcal sexiste et la manifestation d'un rapport de pouvoir alimenté par des relations conflictuelles entre les classes de sexe. Enfin, alors que les égalitaristes s'en prennent aux rôles plutôt qu'aux structures, les radicales revendiquent l'abolition des

24 Retenons, parmi les textes fondateurs: Kate Millett, La politique du mâle, Paris, Stock, 1971 (édition américaine, 1970); Robin Morgan (dir.), Sisterhood is Powerful, New York, Vintage Books, 1970; les articles parus dans le numéro spécial de la revue Partisans, nos 54-55, juillet-octobre, 1970; Shulamith Firestone, La dialectique du sexe, Paris, Stock, 1972 (édition américaine, 197 1); Juliet Mitchell, L'âge des femmes, Paris, Éditions des Femmes, 1974, (édition américaine, 1971), Nicole-Claude Mathieu, «Notes pour une définition sociologique des catégories de sexe», Épistémologie sociologique, no 11, 1971, p. 19-39; Christine Delphy, «Nos amis et nous. Les fondements cachés de quelques discours pseudo-féministes», Questions féministes, vol. 1, no 1, novembre 1977, p. 21-50.

25 Sylvie Chaperon, «La deuxième Simone de Beauvoir», Les Temps modernes, 1996, p. 112. De Simone de Beauvoir, les radicales retiennent surtout l'idée que la hiérarchisation sexuelle, comme mode d'organisation des rapports sociaux, ne pourra être abolie qu'à partir du moment où les femmes seront libérées de leurs fonctions biologiques et des contraintes du maternage. Elles rejettent l'ambiguïté que présente la perspective naturaliste du Deuxième sexe selon laquelle les hommes possèdent une transcendance liée à leurs capacités et activités créatrices, alors qu'une telle «conscience de soi» et de l'histoire ne serait accessible aux femmes qu'au prix de leur disparition au rofit de l'Autre.

26 Ginette Castro, ouvrage cité, p. 282.

27 Par contre, en dépit de leurs positions théoriques divergentes, les féministes radicales formeront souvent des alliances stratégiques avec les féministes égalitaires afin de bénéficier du pouvoir de pression de celles-ci pour revendiquer certaines réformes nécessaires à l'élimination des conditions de la discrimination et à l'obtention du droit à l'autodétermination réclamé par toutes. 
institutions patriarcales pour en finir avec le déterminisme biologique et concrétiser leurs visées libératrices.

L'appellation «radicale» est une appellation aux contours larges et instables. Appliquée à la pensée féministe, elle renvoie à quatre grandes tendances principales: le féminisme matérialiste, le féminisme socialiste, le féminisme de la spécificité ou autonomiste et le féminisme lesbien. Dans la problématique féministe matérialiste, la classe des hommes est expressément nommée par Christine Delphy comme ennemi principal ${ }^{28}$, alors que l'interrelation entre les ordres patriarcal et capitaliste fait l'objet de l'analyse du féminisme socialiste. L'une et l'autre tendance dénoncent l'occultation et l'appropriation de la valeur économique du travail productif des femmes ${ }^{29}$ et s'intéressent à la matérialité de l'oppression individuelle et collective des femmes ${ }^{30}$. Pour leur part, les théoriciennes du courant de la spécificité, que l'on nomme aux États-Unis women centered, plaident en faveur d'une conception autonomiste des luttes des femmes. Elles situent le principe de l'oppression commune et spécifique des femmes dans l'institution familiale ${ }^{31}$ et, plus particulièrement, dans l'obligation et la gratuité du travail domestique ${ }^{32}$. Leur champ d'observation englobe l'étude de la culture de l'oppression et de l'éthique des femmes, telles qu'elles s'expriment à travers les représentations, les comportements et les pratiques de la maternité ${ }^{33}$, et l'analyse des relations mères-filles ${ }^{34}$. Enfin, le féminisme radical lesbien voit dans la contrainte à l'hétérosexualité la manifestation de l'acculturation au pouvoir et à la sexualité Mâles ${ }^{35}$. Il pose le lesbianisme comme choix politique au sein du Questions féministes, no 2, février 1978, p. 5-30; Paola Tabet, «Fertilité naturelle, reproduction
forcée», dans Nicole-Claude Mathieu (dir.), L'arraisonnement des femmes: essais en anthropologie des sexes, Paris, École des hautes études en sciences sociales, 1985, p. 61-147.

31 Adrienne Rich, Naître d'une femme, la maternité en tant qu'expérience et institution, Paris, Denoël-Gonthier, 1986 (édition américaine, 1976).

32 Collectif les Chimères, Maternité esclave, Paris, Union générale d'éditions, coll. 10/18, 1975; Collectif l'insoumise (Genève), Le foyer de l'insurrection, Carouge (Suisse), Collectif l'insoumise, 1977; Natalie V. Sokoloff, Between Money and Love. The Dialectics of Women's Home and Market Work, New York, Praeger, 1980.

33 Joyce Trebilcot (dir.), Mothering: Essays in Feminist Theory, Totowa (N.J.), Rowman and Allan, 1983; Mary O'Brien, La dialectique de la reproduction, Montréal, Éditions du remue-ménage, 1987 (édition américaine, 1981); Nancy Chodorow, The Reproduction of Mothering, Psychoanalysis and the Sociology of Gender, Berkeley, University of California Press, 1978.

34 Nancy Friday, Ma mère, mon miroir, Paris, Laffont, 1979 (édition américaine, 1977); Luce lrigaray, Le corps à corps avec la mère, Paris, Éditions de la Pleine Lune, 1981; Jane Gallop, The Daugther's Seduction: Feminism and Psychoanalysis, Itaca, Cornell University Press, 1982.

35 Parmi un nombre considérable de travaux, citons: Barbara Love et Sydney Abbot, Sappho was a Right-on Woman: A Liberated View of Lesbianism, New York, Stein and Day, 1972; 
féminisme et propose un travail théorique sur le concept de sororité dans le cadre du continuum lesbien.

Cela étant dit, les différentes tendances radicales se rencontrent et se complètent dans leur dénonciation de la société patriarcale. Leurs discours rompent avec tout idéal du «mêmeté» et dénoncent la naturalisation de la différence entre les sexes comme fondement de l'oppression. L'argument prédominant est que les femmes sont opprimées et exploitées individuellement et collectivement en raison de leur identité sexuelle. Le courant radical postule qu'il n'y a pas de domaine privé dans une existence personnelle qui ne soit politique et accorde la primauté aux luttes des femmes. Ainsi, le questionnement radical s'intéresse en particulier aux multiples manifestations sociales de l'oppression-exploitation des femmes; aux rapports d'interdépendance entre reproduction biologique et reproduction sociale; à l'arbitraire de la division sociale en domaines public et privé et à la dévalorisation«invisibilisation» du travail, marchand et non marchand, produit par les femmes.

$\mathrm{Au}$ risque, encore une fois, de trop simplifier, j'ai regroupé sous l'appellation féminisme radical les diverses problématiques qui, dans la recherche d'une théorie générale des rapports de sexe, ont eu pour prémisses communes l'identification du patriarcat comme système socio-économico-politique d'appropriation des femmes et la reconnaissance de l'existence d'une classe de femmes: les femmes constituent une classe politique dans la mesure où elles sont individuellement et collectivement exploitées et opprimées par la classe des hommes, à des fins de production et de reproduction. Par ailleurs, cette même appellation englobe également la critique épistémologique féministe qui a mené à un réexamen critique des présupposés et des critères de la méthode scientifique.

Pour les féministes radicales, il s'impose à l'évidence que les grands récits qui prétendent représenter tout le spectre de l'expérience humaine traduisent pour l'essentiel la suprématie masculine. En découle l'impérieuse nécessité de démarquer, d'éloigner la recherche féministe d'un savoir-faire et d'un savoir-dire androcentristes. Rapport de cause à effet, le féminisme radical incite à rompre avec l'objectivisme de la science moderne qui sépare théorie et action et présuppose que le chercheur peut et doit - se dégager du monde des valeurs, de la subjectivité et de l'expérience. Il sera longuement argumenté que la validité et la légitimité de la recherche féministe

Radicalesbians, «The Woman-Identified Woman», dans Anne Koedt et autres (dir.), Radical Feminism, New York, Quadrangle Books, 1973, p. 240-245; Jill Johnson, Lesbian Nation, The Feminist Solution, New York, Simon \& Schuster, 1974; Monique Wittig, «On ne naît pas femme», Questions féministes, no 8, 1980, p. 75-84; Adrienne Rich, «La contrainte à l'hétérosexualité ou l'existence lesbienne», Nouvelles questions féministes, no 1, mars 1982, p. $15-43$. 
reposent non pas sur l'objectivité ou la neutralité de ses méthodes, mais bien sur la reconnaissance par la chercheuse ou le chercheur de sa position située ${ }^{36}$ et de sa capacité à reconnaître les dimensions hiérarchisées et institutionnalisées des rapports de sexe. Il s'agit en l'occurrence de proposer des modèles d'analyse qui intègrent les femmes comme catégorie sociologique et de s'appuyer sur leur point de vue, et celui de leur monde quotidien ${ }^{37}$, pour mettre fin à la représentation tronquée de la société et des rapports sociaux que reproduisent les sciences sociales.

Autrement dit, il s'agit de rompre avec les habitus scientifiques dominants. L'inscription dans les modèles théoriques du sens et de la nature des rapports de sexage de même qu'une relecture de l'expérience des femmes à travers la réalité de leur vie quotidienne sont respectivement proposées comme manière d'y arriver par les féminismes matérialiste et socialiste et les féminismes de la spécificité et lesbien.

Pour plusieurs, les thèmes et les problèmes abordés par le féminisme radical seront perçus comme étrangers au vécu quotidien des femmes ordinaires, puisque sexualité, amour, mariage et famille y sont désignés, dans leur interdépendance, comme lieux institutionnalisés de l'oppression des femmes et de l'appropriation de leur corps et de leur travail. La dimension négative et «négativante» du discours effraie et repousse. Car si, pour les féministes radicales, il s'agit de faire se développer une conscience de classe, l'accent mis sur les effets pervers de ce que Kate Millet avait nommé l'hétérosexualité pervertie - violence conjugale, inceste, pornographie, mutilations sexuelles, dépendance affective et sociale, etc. - donne à plusieurs l'impression qu'une telle problématique fait peu de place, en dépit des intentions de départ, au vécu quotidien des femmes ordinaires et les relègue a un statut de victimes. Pour d'autres, le radicalisme ne parvient pas ou se refuse à poser la question des besoins ordinaires et essentiels des femmes, des hommes et des enfants et évacue, par le fait même, toute réflexion constructive sur l'identité féminine et la différence. Le féminisme radical se révèle impuissant, constate Geneviève Fraisse ${ }^{38}$, à déplacer l'alternative ancienne devant laquelle les femmes sont tiraillées entre l'assimilation à l'autre sexe et la surdétermination de la différence. Pour son insistance militante à privilégier les notions de classe de femmes, de communauté de sort ou de luttes collectives dans ses schémas explicatifs, il se verra aussi reprocher de ne pas accorder suffisamment d'importance à la diversité et à la multiplicité des identités sociales qui constituent les femmes comme sujet social. Bref, le courant radical apparaît incapable de briser la logique de la pensée dualiste en reproduisant un mode d'analyse qui

36 Sandra Harding et Merill B. Hintikka (dir.), Discovering Reality: Feminist Perspectives on Epistemology, Metaphysics, Methodology, and Philosophy of Science, Dordrecht (Hollande), D. Reidel Publishing, 1983.

37 Dorothy E. Smith, «Le parti pris des femmes», dans Yolande Cohen (dir.), Femmes et politique, Montréal, Le Jour, coll. «Idéelles», 1981, p. 139-147.

38 Geneviève Fraisse, «Entre égalité et liberté», dans Ephesia, ouvrage cité, p. 387-393. 
semble encore concourir à enfermer les femmes dans un système clos de relations sociales. Ces critiques annoncent la toile de fond sur laquelle s'élaboreront les débats des deux dernières décennies au sein des études féministes.

Mais, pour l'instant, retenons que l'approche féministe radicale a eu le grand mérite de mettre en évidence les caractères spécifiques de l'oppression des femmes, de la situer et de déconstruire les arguments naturalistes et culturalistes invoques depuis les temps immémoriaux pour légitimer les rapports de pouvoir entre les sexes. Elle aura largement contribué à remettre en cause les prétentions universalistes de tout savoir construit sur l'exclusion des femmes. Plus concrètement, en rupture avec les interprétations dominantes, elle aura permis de voir, à travers la conceptualisation du patriarcat, de la division sexuelle du travail et de la dichotomie privé/public, que le véritable problème, ici comme ailleurs, est la domination et non la différence entre les sexes ${ }^{39}$. Elle aura également mené à interpréter les violences faites aux femmes non plus comme un problème individuel ou psychosocial, mais bien comme un mécanisme de régulation sociale, comme un moyen de contrôle social. Ces contributions ont été déterminantes pour briser la loi du silence touchant des sujets jusqu'alors tabous et remettre dans une perspective sociétale et dialectique la vie sexuelle, affective, familiale, économique et socio-politique des femmes. Enfin, le concept de patriarcat aura été d'une importance politique capitale pour inscrire la lutte des femmes comme lutte sociale au même titre que les luttes menées par les autres grands mouvements sociaux ${ }^{40}$.

39 Catherine MacKinnon, Feminism Unmodified: Discourses on Life and Law, Cambridge (Mass.), Harvard University Press, 1987; Anne-Marie Daune-Richard et Anne-Marie Devreux, art. cité, p. 7-30; Marie-Claude Hurtig, Michèle Kail et Hélène Rouch (dir.), Sexe et genre. De la hiérarchie entre les sexes, Paris, CNRS, 1991.

40 Bonnie J. Fox, «Conceptualizing "Patriarchy"», Revue canadienne de sociologie et d'anthropologie, vol. 25, no 2, 1988, p. 163-182. 


\section{c) La réconciliation du féminin et du maternel}

\section{$\underline{\text { Retour à la table des matières }}$}

La conjoncture du début des années quatre-vingt entraîne la mise en veilleuse de la plupart des thèses collectivistes au profit de la redécouverte de l'individualisme, d'un retour sur le privé et de la valorisation de la qualité de vie. Dans cette conjoncture, la théorie féministe radicale perd de son autorité comme modèle d'interprétation et lieu de mobilisation politique. D'autant plus que, encouragées par des gains substantiels réalisés dans la sphère publique, plusieurs femmes occidentales laissent savoir qu'elles souhaitent dorénavant éviter tout nouveau soubresaut qui risquerait de déstabiliser leur manière d'être à deux et de vivre leur maternité: leur rêve d'harmonie et de stabilité à l'«ère de l'éphémère» cohabite difficilement avec l'opposition radicale. Questions accessoires dans le cadre de l'analyse égalitariste, niées dans les formes les plus agressives du féminisme radical, la maternité, l'identité et la différence ${ }^{41}$, puis, éventuellement, les rapports hommes-femmes ${ }^{42}$ se hissent ainsi au rang de thèmes premiers du néo-féminisme.

Pour les unes, la conscience d'avoir marginalisé sinon gommé leur vécu de femme et de mère les incite à jeter un pont entre un radicalisme dénonciateur et un modèle égalitariste réducteur afin de se réapproprier leur expérience de femme et de mère et la problématiser dans une certaine cohérence par rapport à leur vécu individuel et collectif. Inquiètes de voir autant de femmes condamnées au surmenage, à la pauvreté et à l'exclusion, les féministes néo-égalitaristes sont à la recherche d'un «second souffle ${ }^{43} \gg$. Sur fond de «ras-le-bol» des super-femmes et de diagnostic de crise de la famille et du couple, plusieurs s'insurgent contre l'inefficacité et la partialité des

41 Anne-Marie De Vilaine, Laurence Gavarini et Michèle Le Coadic (dir.), Maternité en mouvement: les femmes, la re/production et les hommes de science, Montréal, Éditions Saint-Martin, 1986, p. 17-21; Marisa Zavalloni (dir.), L'émergence d'une culture au féminin, Montréal, Éditions Saint-Martin, 1987; Margaret A. Simon, «Motherhood, feminism and identity», Women's Studies International Forum, vol. 7, no 5, 1984, p. 349-369; «Le féminisme une redéfinition des identités», dans Ephesia, ouvrage cité, partie 5, p. 328-369.

42 Parmi les premiers écrits qui revisitent le sujet, mentionnons de: Geneviève Delaisi de Parseval et Alain Janaud, La part du père, Paris, Seuil, 1981; Annie Leclerc, Hommes et femmes, Paris, Bernard Grasset, 1985; Elizabeth Badinter, L'un est l'autre. Des relations entre hommes et femmes, Paris, 0. Jacob, 1992.

43 Betty Friedan, Femmes: le second souffle, Montréal, Éditions Stanké, 1983 (édition américaine, 1976). 
réaménagements survenus dans les rôles de sexe. Elles dénoncent l'incapacité du monde du travail à tenir ses promesses d'autonomisation et de réalisation de soi et suggèrent de réévaluer avec pragmatisme certaines des valeurs qui ont été mises de l'avant, tout comme les choix qui ont été posés ${ }^{44}$. D'autres adoptent plutôt le ton de la motivation et de l'optimisme pour inviter les femmes à prendre conscience de leur pouvoir et les inciter à en user de manière positive dans leurs négociations personnelles, professionnelles et politiques ${ }^{45}$. Enfin, dans le contexte des discussions sur l'à-propos du principe de parité ou des programmes d'accès à l'égalité, plusieurs s'intéressent aux contradictions et problèmes que soulève la persistante disparité entre l'égalité formelle et l'égalité de fait, particulièrement dans les sphères professionnelle et politique ${ }^{46}$. Se demandant si les femmes ont été prises au «piège de l'égalité», elles engagent le débat sur l'opportunité d'appliquer des mesures différentielles ou de discrimination positive pour compenser les effets négatifs toujours présents de la discrimination systémique. Dans cette optique, Okin ${ }^{47}$ remarque que la question cruciale à élucider au sujet de la loi -comme en ce qui concerne toute institution sociale - n'est pas tant de savoir si celle-ci traite ou devrait traiter les femmes et les hommes différemment ou de la même manière, mais bien de savoir si elle «encourage la domination des hommes et le désavantage des femmes ${ }^{48}$ ». Entre ces pôles de réflexion, un consensus stratégique se dégage pour réclamer l'atténuation, dans l'immédiat, des contraintes de la double tâche, dont le poids est encore largement porté par les femmes, et l'instauration de mesures de soutien à la famille et aux trajectoires professionnelles.

Pour d'autres, qui choisissent d'abandonner la problématique de l'égalité ou l'opposition à caractère socio-politique, il s'agit plutôt d'offrir à chaque femme les voix/voies de sa libération pour faire contrepoids au silence obscurantiste de la psychanalyse sur le sujet féminin et au refus militant des radicales de problématiser le territoire féminin. Elles s'intéressent plus aux problèmes d'éthique et d'identité du

44 Germaine Greer, Sexe et destinée, Paris, Grasset, 1986 (édition anglaise, 1984); Virginia Held, «The obligations of mothers and fathers», dans Mary Vetterling-Braggin (dir.), Feminity, Masculinity and Androgyny, New Jersey, Rowman and Littlefield, 1982; Jean Bethke Elshtain, Public Man, Private Woman: Women in Social and Political Thought, Princeton, Princeton University Press, 1981.

45 Le best-seller de Naomi Wolf, Fire with Fire (Toronto, Random House, 1993), est exemplaire à cet égard.

46 Un dossier intéressant sur cette question est présenté par la revue Nouvelles questions féministes dans un numéro spécial Parité - Pour, vol. 15, no 4, 1994. Voir également le chapitre «Égalité, différences, rapports de pouvoir», dans Ephesia, ouvrage cité, p. 375-406.

47 Susan Moller Okin, «Sur la question des différences», dans Ephesia, ouvrage cité, p. 60.

48 Le livre de Drucilla Cornell, Beyond Accomodation. Ethical Feminism, Deconstruction, and the Law (New York, Routledge, 1991), s'intéresse à cette question de façon magistrale. 
sujet féminin qu'à la dimension sexuée des rapports sociaux ${ }^{49}$. Souvent en situation d'extériorité par rapport aux pratiques et à l'action féministes, ces nouvelles paroles, elles-mêmes éclatées et multiples, proposent une réflexion épistémologique sur la différence, le genre, la féminitude et le féminin. Constituant en quelque sorte le refoulé des discours féministes antérieurs, elles envahissent un large espace de théorisation avec des discussions sur le sujet féminin, le corps, la gestation, l'enfantement et la jouissance maternelle, vus comme espaces distinctifs et lieux premiers de la différence et de la relation à l'Autre. Les théoriciennes de ce courant que j'ai appelé féminisme de la fémelléité ${ }^{50}$ proposent comme solution de rechange une théorie du féminin-maternel qui privilégie la réappropriation du territoire et de l'imaginaire féminins propres a l'expérience du corps sexué et de l'enfantement. Dans un contexte où, par ailleurs, l'essor des nouvelles technologies de reproduction bouleverse les assises mêmes du rapport des femmes à la procréation, leurs discours réintroduisent l'idée de la maternité comme lieu de la rencontre entre la nature et la culture. Les théoriciennes fémelléistes y voient là l'espace identitaire distinctif des femmes et la source cognitive d'une éthique féminine en rupture avec les logiques productiviste et techniciste.

En nommant le féminin-maternel comme voie de libération et contre-pouvoir à protéger de l'emprise patriarcale et de l'assujettissement aux valeurs marchandes, les tenantes de ce courant expriment non seulement leur incrédulité à l'égard des discours égalitaristes et radicaux, mais encore leur crainte d'avoir été piégées, niées par les revendications mises de l'avant par le mouvement des femmes. Plusieurs d'entre elles se montrent plus que sceptiques quant à l'intérêt de poursuivre le dialogue avec les discours et savoirs dominants dans la course à la productivité et au pouvoir social. Elles craignent en outre que le refus de s'identifier au destin maternel et la conceptualisation du travail salarié comme voie d'émancipation ne privent les femmes de leurs savoirs spécifiques et de leur singularité, autrement dit de leur identité même. Enfin, elles soupçonnent que l'insistance radicale à désigner la famille et le maternage comme lieux de l'oppression économique, sexuelle et psychique des mûres mènera, à l'instar de la pensée rationaliste moderne, à l'effondrement de l'éthique féminine. Inquiétudes qui, il va sans dire, sont au coeur d'une quête d'un sens féminin du soi. Inquiétudes qui, par ailleurs, non seulement sont étrangères à la détermination des conditions objectives réelles de dépendance et d'infériorité vécues par les femmes dans leurs rapports institués à la maternité et au couple, mais encore qui ne s'accompagnent d'aucune visée de transformation de la dynamique des rapports sociaux sexués.

49 Parmi les textes majeurs, soulignons celui de Mary Daly, Gyn/Ecology: The Metaethics of Radical Feminism, Boston, Beacon Press, 1978, et celui de Luce Irigaray, Éthique de la différence sexuelle, Paris, Minuit, 1984.

50 Voir l'introduction et la note 20. 
Il ne faudrait pas donner ici l'impression qu'il y a une homogénéité de pensée parmi les auteures que j'ai regroupées sous le vocable de fémelléistes. L'évolution multiple du discours féministe au cours des années quatre-vingt exigerait des distinctions plus fines. Je me contenterai de distinguer les deux tendances qui, au cours de la décennie suivante, seront passées au crible tant par les militantes que par les intellectuelles féministes. La première réunit les penseuses de la différence parmi lesquelles se retrouvent plusieurs Américaines ${ }^{51}$. La seconde, que les critiques américaines des années quatre-vingt-dix qualifieront abusivement de french feminism et associeront, sans les nuances nécessaires, au courant radical, se développe parmi les protagonistes de la critique psychanalytique et littéraire française ${ }^{52}$.

Les travaux des féministes de la différence se situent dans la continuité de la réflexion développée principalement aux États-Unis a l'intérieur de la tendance radicale de la spécificité ${ }^{53}$. Mais si cette dernière souscrivait au déni radical de la différence et à la dénonciation des institutions patriarcales, une tout autre conception informe la plupart des travaux du courant de la différence, à savoir que les femmes seraient porteuses d'une culture autre, d'un nouveau projet social ${ }^{54}$. Leur réponse est sans équivoque. S'inspirant nommément de la thèse de Nancy Chodorow ${ }^{55}$, les féministes de la différence soutiennent que les femmes, en raison même de leur assignation à la sphère domestique et de leur rôle de parent principal, ont développé un sens du soi fondamentalement localisé (rapport au corps), entouré et relationnel (souci de l'Autre). A l'opposé, le sens masculin du soi serait, lui, construit dans l'abstrait, séparé du monde et de l'Autre. Poussant plus loin l'argument, Sara Ruddick ${ }^{56}$ soutiendra que la quête de liberté est un piège si elle signifie l'adoption du modèle masculin du soi, de la raison et du progrès: l'action politique exige au contraire d'exploiter le potentiel subversif des valeurs féminines plutôt que d'endosser

51 Sont représentatifs de cette approche les travaux de Sara Ruddick, «Maternal thinking», Feminist Studies, vol. 6, no 2, été 1980, p. 342-367, de Jean Bethke Elshtain, ouvrage cité, et de Carol Gilligan, Une si grande différence, Paris, Flammarion, 1986 (édition américaine, 1982).

52 Parmi les auteures les plus connues de cette tendance, on retiendra les noms de Luce Irigaray, Speculum de l'autre femme, Paris, Minuit, 1974, et Ce sexe qui n'en est pas un, Paris, Minuit, 1977; d'Hélène Cixoux, «Le rire de la Méduse», L'Arc, 1975, no 61, p. 39-54; et de Julia Kristeva, Histoire d'amour, Paris, Denoël-Gonthier, 1983.

53 Voir les notes 33 et 34. En France, les travaux de Sonia Dayan-Herzbrun, «La grève des ventres» (Connexions, no 27, 1979, p. 85-100) et «Production du sentiment amoureux et travail des femmes» (Cahiers internationaux de sociologie, vol. 72, janvier-juin 1982, p. 113-130), s'inscrivent dans cette tradition.

54 Cette question constitue le fil conducteur des essais publiés dans Anne-Marie De Vilaine, Laurence Gavarini et Michèle Le Coadic (dir.), ouvrage cité, et dans Marisa Zavalloni (dir.), ouvrage cité.

55 Nancy Chodorow, The Reproduction of Mothering, Psychoanalysis and the Sociology of Gender, Berkeley, University of California Press, 1978.

56 Sara Ruddick, art. cité. 
un discours qui les invalide. La psychologue Carol Gilligan, dont le livre Une si grande différence se retrouvera dans la liste des best-sellers, suggère quant à elle «de se mettre à l'écoute de la voix différente des femmes», pour concevoir les relations avec autrui, puisque les femmes ont atteint, soutient-elle, un stade de développement moral plus élevé qui «influence leur rapport avec autrui, et leur sens des responsabilités ${ }^{57}$ ». Unissant «le cœur et l'esprit», le jugement moral des femmes relèverait donc d'une éthique plus noble que celle des hommes, puisque fondé sur la «sollicitude» et des relations concrètes avec autrui, plutôt que sur la reconnaissance abstraite et rationalisée des droits, caractéristique de l'éthique masculine.

L'ambition des féministes de la différence est de revaloriser l'éthique féminine à l'œuvre dans le domaine de l'intimité et du domestique et de lui conférer un caractère paradigmatique. Car, estiment-elles, le langage de la modernité s'est reflété non pas tant dans une pratique d'oppression matérielle que dans une opposition systématique et récurrente à la conscience du soi féminin dont les valeurs et les expériences écoute de l'autre, altruisme, générosité, don de soi, amour, tendresse, affectivité, soins, etc. - ont été discréditées sur le plan social et reléguées au domaine du privé. Or cette conscience du soi féminin et la façon d'être en rapport avec l'Autre qui l'accompagne devraient au contraire constituer le fondement même d'une critique de la vision androcentriste de la raison, de la subjectivité, de la justice et de la liberté. Pour offrir une vision créatrice et libératrice de la vie, les féministes de la différence proposent donc de réexaminer les valeurs de l'expérience maternelle et de faire une place à cette «voix différente» des femmes que la culture dominante a toujours cherché à étouffer. La conscience féminine du soi, les «pouvoirs élémentaux des femmes de se remémorer/de se remembrer ${ }^{58}$ », leur manière d'être et de penser autrement ouvriraient alors la voie à la primauté d'une éthique de l'«Étance», de l'équité, de la non-violence et de la responsabilité face à autrui. Une telle éthique, balisée par l'histoire et la culture des femmes, serait alors étrangère à toute impulsion de contrôle et reconnaîtrait à l'Autre sa singularité et son individualité.

Si les féministes de la différence prennent comme point de départ le confinement des femmes à la sphère privée et la dimension relationnelle de leur expérience maternelle pour élaborer leurs propositions théoriques sur l'éthique féminine, les auteures qui appartiennent au courant de la critique psychanalytique et littéraire axent quant à elles leur réflexion sur les dimensions symbolique et métaphorique de l'expérience maternelle. Maternité et rapport à la Mère sont ici définis comme continent noir de la pensée moderne, emprisonné dans l'ombre déformante de la culture binaire phallocentrique et du langage normatif des hommes. Influencées par

Carol Gilligan, ouvrage cité, p. 66.

58 Mary Daly, «Pouvoirs élémentaux des femmes: re-mémoration/re-membrement», dans Marisa Zavalioni (dir.), ouvrage cité, p. 133-146. 
les travaux de Lacan et de Derrida, ces féministes, qu'Iris Young ${ }^{59}$ a qualifiées de «gynocentristes», se concentrent sur le langage et l'ordre symbolique pour requestionner le féminin et l'espace maternel comme lieux de la différence et de l'Être-femme. La maternité y devient acte de création et l'accent est mis sur le potentiel procréateur-créateur des femmes comme source de pouvoir et d'identité. C'est surtout dans la littérature française, paradoxalement plus souvent citée par des universitaires américaines ${ }^{60}$, que la métaphore prendra une importance sans Précédent comme langage de libération du féminin. Penser le féminin, même en dehors de tout rapport social concret, devient action d'affirmation identitaire. La voie de libération dont il est ici question est circonscrite aux domaines des idées et de l'Être-femme. Elle vise la réalisation d'un soi «psychique» par la réappropriation de l'inconscient, du territoire et de l'imaginaire féminins propres à l'expérience de la maternité, sans référence aucune a une proposition collective de libération des femmes.

Les auteures qui se rattachent à ce courant choisissent de travailler dans une perspective déconstructiviste pour retrouver l'Autre, le corps sexué, l'Absente du langage. Plusieurs s'engagent dans une démarche pour imaginer une théorie du sujet qui ne soit pas appropriée par le masculin et une écriture intégrale ${ }^{61}$ à travers laquelle les femmes pourraient s'identifier et se réapproprier leur imaginaire et leur identité. Leur projet est celui d'une écriture du langage du corps qui permette de faire resurgir les traces du féminin-maternel refoulé ${ }^{62}$ qui se tait et se terre, et de le déchiffrer hors $\mathrm{du}$ «parler homme ${ }^{63}$ ». Pour ces auteures,. remarque Céline Séguin, «le fait d'être le "deuxième sexe" ou de représenter l'Autre - l'absente, la marginalisée, la rejetée, l'exclue, l'abandonnée, le refoulé, l'immanent - ne constitue plus une condition à transcender. Le féminin représente de façon métaphorique l'Autre et cette altérité, plus qu'une condition d'infériorité, devient un mode de vie, de pensée et d'écriture qui permet l'ouverture, la pluralité, la diversité, la différence, qui permet donc le

59 Iris Marion Young, «Humanism, gynocentrism and feminist politics», Women's Studies International Forum, vol. 8, no 1, 1985, p. 173-183.

60 Eleanor H. Kuykendall, «Subverting essentialism», Hypatia, vol. 6, no 3, 1991, p. 208-217; Donna C. Stanton, «Difference on trial: A critique of the maternal metaphor in Cixous, Irigaray, and Kristeva», dans Nancy K. Miller (dir.), The Poetics of Gender, New York, Columbia University Press, 1986, p. 157-182; Elizabeth Gross, «Philosophy, subjectivity and the body: Kristeva and Irigaray», dans Carole Pateman et Elizabeth Gross (dir.), Feminist Challenges. Social and Political Theory, Boston, Northeastern University Press, 1986, p. 125-143.

61 Nicole Brossard, «De radical à intégrales», dans Marisa Zavalloni (dir.), ouvrage cité, p. 163-174; Luce Irigaray (dir.) «Le sexe linguistique», Langages, no 85, 1987, et Le temps de la différence. Pour une révolution pacifique, Paris, Librairie générale française, Le Livre de Poche, 1989.

62 Isabelle Lasvergnas-Grémy, «La trace du féminin dans la pensée? Quelques échos des débats contemporains sur l'altérité», Cahiers de recherche sociologique, vol. 4, no 1, 1986, p. 115-137.

63 Luce Irigaray, Éthique de la différence sexuelle, ouvrage cité, 1984, p. 25. 
dépassement de la pensée binaire ${ }^{64} \gg$. Articulé à une réflexion sur le corps et la sexualité des femmes comme expérience de la multiplicité, «puisque la femme a des sexes un peu partout ${ }^{65}$, ce discours de la fémelléité cherche à réfuter l'éloge du même et de la primauté de la raison instrumentale caractéristique de la tradition philosophique occidentale. Plusieurs des auteures à l'origine de la pensée fémelléiste choisiront d'ailleurs au fil des ans de prendre, plus ou moins explicitement, leurs distances par rapport au féminisme ${ }^{66}$. Elles lui reprochent surtout d'être porteur d'une logique formelle qui tend à stabiliser, à tout réorganiser, à rationaliser et à uniformiser plutôt qu'à encourager la prise en compte de l'instable, du diffus et de la différence.

Par une glorification du féminin-maternel comme métaphore de l'altérité, les chantres du fémelléisme gynocentriste cherchent à s'abstraire d'une logique de l'assimilation et de la négation de la plénitude du corps sexué féminin. Il leur importe d'amener les femmes a se définir elles-mêmes, à travers leur propre imaginaire, corporalité et expérience, plutôt qu'à travers le miroir déformant des normes et des références investies par le sujet masculin. Le projet séduit. Leur discours valorise les qualités et les aptitudes du féminin, ordinairement décriées. Okin y voit «un heureux antidote à la surestimation des hommes par le premier féminisme ${ }^{67}$ ». À mon avis, un tel discours a l'intéressant mérite de prendre en considération l'apport possible d'une réflexion sur la culture féminine nourricière à la philosophie et à la subversion des concepts de raison, d'humanisme et de progrès.

Mais la difficulté que pose le courant fémelléiste est sérieuse. Il exalte, sinon resacralise, un féminin-maternel immuable, essentiellement inscrit dans le corps, comme lieu et métaphore de l'altérité et de contrepouvoir, sans préciser les multiples façons dont les sociétés se représentent ce féminin-maternel, en usent et en abusent, «sans préciser non plus les contextes sociaux des représentations symboliques invoquées ${ }^{68}$ » et la diversité des contraintes matérielles et institutionnelles inhérentes à l'expérience quotidienne de la maternité. Un Féminin et un Masculin invariables, permanents, sont ainsi figés sans possible rapprochement de leur destin biologique, leur univers normatif et leurs représentations symboliques.

Fort différentes l'une de l'autre, les deux tendances du féminisme de la fémelléité dont il a été ici question se rejoignent néanmoins dans la mesure où elles considèrent que le discours scientifique et le système de valeurs articulés autour du sujet rationnel

64 Céline Séguin, Féminisme et post-modernisme: vers une alternative féministe post-moderne?, texte polycopié, 1992 , p. 45.

65 Luce Irigaray, Ce sexe qui n'en est pas un, ouvrage cité, p. 28.

66 Sous ce rapport, on pensera en particulier à Julia Kristeva et Hélène Cixous.

67 Susan Moller Okin, art. cité, p. 58.

68 Joan W. Scott, «Genre: une catégorie utile d'analyse historique», Les Cahiers du GRIF, nos 37-38, 1987, p. 125-153. 
procèdent d'un univers masculin qui s'oppose à l'ethos du féminin. L'une et l'autre posent le problème de la relation entre identité et culture. L'une et l'autre théorisent les rapports sociaux de sexe en dehors de leurs répercussions matérielles et socio-politiques bien concrètes. Pour contrecarrer l'acculturation des femmes à un mode de pensée qui les exclut, à un langage et un imaginaire qui leur sont étrangers, la première cependant entend revaloriser, comme expression éthique du féminin, la manière maternelle d'entrer en relation et de s'occuper des autres, enfants et adultes confondus ${ }^{69}$, alors que la seconde s'attarde essentiellement à la construction d'un sujet féminin défini par sa seule expérience symbolique de la maternité et son expression esthétique dans l'acte de création et d'écriture au féminin. Certes, dans les deux discours, la maternité est réhabilitée comme espace et territoire du féminin. Mieux, elle est réifiée en pouvoir dans un langage plus proche de la poésie que de la démonstration. Cependant, si l'un et l'autre discours donnent rapidement lieu à une interprétation essentialiste du féminin, il importe de remarquer que la problématique développée par les féministes de la différence relève plus d'une interprétation culturaliste que d'une interprétation essentialiste: la spécificité féminine, ou, devrait-on dire, la spécificité des femmes à laquelle elles se réfèrent est moins celle de leur expérience intime et immédiate de leur corps sexué que le produit historique de relations sociales sexuées et de rapports institutionnalisés à la maternité.

En dépit de leur pouvoir évocateur, de leurs intonations lyriques, ces discours relèvent d'une lecture qui ne tient pas compte des aspects historique et social. Le féminisme gynocentriste, dans ses expressions plus excessives, suggère une hypothétique suprématie des valeurs féminines ou, plus prosaïquement, le remplacement des valeurs masculines par les valeurs féminines. Quant au discours du féminisme de la différence, il se situe pour sa part hors du temps et de tout espace réel. Il exclut toute évocation matérielle de la réalité dynamique, mutante et sexuée dans laquelle se pensent et s'actualisent la féminité et la relation concrète et quotidienne avec l'enfant, avec ses incitations et ses contraintes, ses paradoxes et ambiguïtés.

Qui plus est, les penseuses du courant de la fémelléité procèdent à un recouvrement entre féminin et maternité qui conduit immanquablement à une nouvelle conception de la féminitude du sujet féminin à partir de sa seule identité maternelle. Une telle conception débouche sur cet autre non-sens qui consiste à définir toutes les femmes comme des mères et à confondre, sinon à assimiler, au nom de leur capacité d'enfanter, la réalité, les besoins et les intérêts des premières à ceux

69 Margaret A. Simons, <<Motherhood, feminism and identity $>>$, Women's Studies International Forum, vol. 7, no 5, 1984, p. 349-359. 
des secondes ${ }^{70}$. Pour Jane Flax ${ }^{71}$, les thèses développées au sein du féminisme de la différence reposent non seulement sur une conception réductrice de la diversité et de l'hétérogénéité des expériences vécues par les femmes, mais également sur une vision idéalisée de la maternité, du maternage et de l'intimité qui ne tient pas compte des multiples tensions, contradictions et contraintes qui marquent le vécu maternel des femmes et leur rapport à la famille, aux enfants ou au conjoint. Largement essentialiste, le féminisme de la fémelléité ne véhicule pas non plus de projet susceptible d'inciter les femmes à s'engager sur le terrain du politique pour améliorer leurs conditions de vie. Au contraire, par son utilisation constante de la métaphore pour exprimer le sujet-femme et son éthique, il rend très difficile la compréhension des réalités historiques, sociales et quotidiennes des rapports de sexe ${ }^{72}$ qui ont été supprimés, déformés ou nies par les discours et savoirs androcentristes dominants.

\section{II. \\ La fin des grands courants: vers un féminisme solidaire}

$\underline{\text { Retour à la table des matières }}$

Ainsi, au gré des ans, de parole relativement unifiée, le discours féministe se morcelle, se fragmente du point de vue de ses intentions et de ses finalités. Les projets théoriques et politiques du féminisme se diversifient, tandis que ses conceptions de l'égalité et de la libération se multiplient et se complexifient ${ }^{73}$. L'idéal démocratique

70 Christine Delphy, «Libération des femmes ou droits corporatistes des mères», Nouvelles questions féministes, nos 16-17-18, 1991, p. 93-118. Pour une plus ample analyse des différentes problématiques de la maternité développées dans des études féministes, voir Evelyn Nakno Glenn, «Social constructions of mothering: A thematic overview >>, dans Evelyn Nakano Glenn, Grace Chang et Linda Rennie Forcey (dir.), Mothering. Ideology, Experience, and Agency, New York, Routhledge, 1994, p. 1-32, et Francine Descarries et Christine Corbeil, «Entre discours et pratiques: l'évolution de la pensée féministe sur la maternité depuis 1960», Nouvelles questions féministes, vol. 15, no 1, 1994, p. 69-93.

71 Jane Flax «Postmodernism and gender relations in feminist theory», Signs, vol. 12, no 4, 1987, p. 621-643. Cet article a été reproduit en diverses versions dans différents recueils de textes portant sur la relation entre féminisme et post-modernisme.

72 Maryse Guerlais, «Vers une nouvelle idéologie du droit statutaire: "Le temps de la différence de Luce lrigaray"», Nouvelles questions féministes, nos 16-17-18, 1991, p. 63-92.

73 Huguette Dagenais (dir.), Science, conscience et action. 25 ans de recherche féministe au Québec, Montréal, Éditions du remue-ménage, 1996. 
du «même» à l'origine du courant du féminisme égalitaire se voit continuellement remis en question par la hantise du «pareil au même». À l'autre pôle, l'essentialisme plus ou moins explicite du féminisme de la fémelléité se voit questionné par toutes celles qui refusent de se laisser enfermer dans une définition singulière, statique et apolitique «d'une identité de genre dont il [le mouvement féministe] conteste précisément la permanence ${ }^{74} \gg$ et les contraintes instituées. L'atteinte d'une unité dans la parole des femmes, de même que leur mobilisation autour d'une théorie commune, qui avait été perçue à l'origine comme l'enjeu théorique et militant du projet féministe, est donc de moins en moins une priorité. Surtout que, dans la foulée des idées contemporaines et de l'expression des singularismes, cette unité et cette mobilisation sont de moins en moins envisagées comme possibles. Les certitudes ont progressivement fait place au doute. Déjà, à la fin des années soixante-dix, la représentativité et le potentiel de rassemblement du mouvement des femmes avaient été mis en doute par les critiques répétées de nombreux groupes ou collectifs de femmes qui se reconnaissaient difficilement dans un projet intellectuel et social principalement pensé et animé par des femmes blanches, hétérosexuelles, appartenant à la classe moyenne. Féministes afro-américaines, collectifs de femmes immigrantes ou autochtones, lesbiennes ou activistes des pays du Sud, pour ne nommer que les plus présentes, reprochent aux théories féministes de passer sous silence leurs situations respectives et de promouvoir un modèle de libération peu adapté aux besoins des femmes de conditions sociales ou de cultures autres. Elles ont été nombreuses 75 à montrer que la seule évocation de conditions communes ou universelles, bien que politiquement efficace pour favoriser la mobilisation initiale, ne

74 Micheline De Sève, «Femmes, action politique et identité», Cahiers de recherche sociologique, no 23, 1994, p. 26.

75 Les références suivantes ne sont que quelques exemples parmi une imposante bibliographie: Margaret A. Simons, «Racism and feminism: A schism in the sisterhood», Feminist Studies, vol. 5, no 2, 1979, p. 384-407; Angela Davis, Women, Race and Class, New York, Vintage Books, 1981; Gloria Joseph, «The incompatible ménage à trois: Marxism, feminism and racism», dans Lydia Sargent (dir.), Women and Revolution, Montréal, Black Rose, 1981, p. 91-107; Gloria Joseph et Jill Lewis, Common Différences: Black and White Feminist Perspectives, Garden City (N.Y.), Anchor Press/Doubleday, 1981; Bonnie Thornton Dill, «Race, class and gender: Prospects for an all-inclusive sisterhood», Feminist Studies, vol. 9, no 1, 1983, p. 131-150; Bell Hooks, Feminist Theory. From Margin to Center, Boston, South End Press, 1984, Evelyn Nakano Glenn, «Racial ethnic women's labor: The intersection of race, gender and class oppression», Review of Radical Political Economics, vol. 17, no 3, 1985, p. 86-108. Plus récemment, Gita Sen et Caren Grown, Femmes du Sud. Autres voix pour le XXIe siècle, Paris, Côté-femmes, 1992. Sur la question lesbienne, voir, entre autres, Monique Wittig, «La pensée straight», Questions féministes, no 7, février 1980, p. 45-53; Line Chamberland, «Le lesbianisme: continuum féminin ou marronnage? Réflexions féministes pour une théorisation de l'expérience lesbienne», Recherches féministes, vol. 2, no 2, 1989, p. 135-146; Shane Phelan, Identity Politics: Lesbian Feminism and the Limits of Community, Philadelphie, Temple University Press, 1989; Sarah Franklin et Jackie Stacey, «Le point de vue lesbien dans les études féministes», Nouvelles questions féministes, nos 16-17-18, 1991, p. 119-136; Christine Holmlund, <<The lesbian, the mother, the heterosexual lover: Irigaray's recordings of difference>>, Feminist Studies, vol. 17, no 2, été 1991, p. 283-308. 
peut plus tenir la route face à l'extrême variabilité des situations vécues par les femmes et des enjeux socio-politiques qu'elles font surgir. La polysémie de concepts, souvent considérés a priori comme féministes et universellement applicables, apparaît alors dans toute son acuité comme matérialisation incontournable de conjonctures culturellement et politiquement situées et contingentes ${ }^{76}$.

Certaines de ces observations seront reprises ultérieurement par les féministes qui, s'inscrivant dans la profonde désillusion post-moderne à l'égard des méta-récits et des grands projets collectifs, reprochent pour leur part aux formulations théoriques et politiques du féminisme occidental leur dépendance à l'endroit des fondements philosophiques et politiques de la modernité et des prémisses des grands récits modernes ${ }^{77}$. Pour ces théoriciennes «des» différences qui préconisent surtout d'incorporer la sensibilité déconstructionniste aux modèles féministes d'analyse, la nécessité d'un travail sur la transformation du sujet politique femme, sur la base d'une plus grande sensibilité à l'altérité, à la diversité, à la multiplicité et à la complexité, s'impose d'elle-même ${ }^{78}$, tandis que celles qui s'identifient plus étroitement à la pensée post-moderne sont réfractaires à «la perspective de proposer la moindre généralisation a propos des femmes ou du genre ${ }^{79}$. Les discussions de l'heure forcent les féministes à se montrer plus attentives à l'interdépendance des divers facteurs de hiérarchie et de division dans leurs tentatives pour expliquer comment et pourquoi les idéologies et les structures patriarcales sont reproduites à travers une multitude de formes et de rapports, tantôt sous prétexte de rationalisme ou d'utilitarisme, tantôt encore sous prétexte de naturalisme ou de relativisme. La pensée féministe contemporaine en vient ainsi à s'interroger de plus en plus sur l'être femme, sur l'être avec l'autre ou les autres. Les notions de différence, d'indifférence, de différences, d'identité et d'égalité s'installent au coeur des débats soulevés par les féministes et par leurs critiques, alors que la réflexion politique se centre peu à peu sur les possibilités de développer et de maintenir une perspective féministe solidaire, ou globale, qui permettrait aux femmes de rester en rapport les unes avec les autres, «pour apprendre les unes des autres malgré la diversité des problèmes et la nécessité de solutions particulières adaptées au contexte ${ }^{80}$ ».

76 La longueur et l'ampleur des négociations requises pour arriver à établir un langage collectivement acceptable dans les instances internationales ou une plate-forme politique commune à l'occasion de conférences internationales, telle la IVe Conférence des Nations unies sur les femmes, à Beijing, en 1995, sont exemplaire des diverses difficultés rencontrées.

77 Chris Weedon, ouvrage cité; Linda J. Nicholson (dir.), ouvrage cité; Jane Flax, Thinking Fragments: Psychoanalysis, Feminism, and Postmodernism in the Contemporary West, Berkeley, University of California Press, 1990.

78 Colette St-Hilaire, art. cité.

79 Susan Moller Okin, art. cité, p. 64.

80 Ibid., p. 65-66. 


\section{Vers un féminisme pluriel, pluraliste et solidaire}

$\underline{\text { Retour à la table des matières }}$

Tensions et contradictions ont toujours existé au sein des courants de pensée du mouvement féministe. Mais si celles-ci ont été éléments de division et d'opposition, elles ont aussi évolué dans un continuum d'interrelations intellectuelles et militantes rendues possibles par la reconnaissance sociologique du rapport de pouvoir constitutif de la division sociale des sexes: rapport de pouvoir traditionnellement établi sur le principe de l'exclusion des femmes de toutes les sphères du social, à l'exception de la famille. En ce sens, il est permis de considérer que ces tensions et contradictions ont contribué à l'enrichissement, voire à la transformation, des modèles initialement proposés. Elles peuvent même être considérées comme des composantes dynamiques du processus critique multidimensionnel qui a marqué l'évolution de la pensée féministe et nourri son ambition de théoriser l'altérité, la diversité et les inégalités à travers les multiples points de vue des femmes, et de faire du combat des droits des femmes celui de tous les opprimés ${ }^{81}$.

Penser le genre comme moyen de «repenser la question de son rapport au sexe ${ }^{82}$ » est dorénavant exploré comme possibilité théorique d'échapper autant à une conception dualiste et immuable des rapports entre les sexes qu'à une interprétation unitaire et globalisante des expériences féminines ${ }^{83}$. Dans cette optique, Scott 84 propose de recourir au concept de genre pour mettre un terme à toute représentation ou interprétation «homogénéisante» du monde des femmes, qu'une telle interprétation s'appuie sur la communauté de leurs intérêts ou de leurs expériences «en tant que femmes» pour développer un point de vue féministe ou qu'elle cherche à retrouver une éthique ou une parole qui leur serait propre. Scott voit dans le genre «une catégorie utile d'analyse historique» non seulement pour que puisse se développer une conscience plus aiguë des différences entre les femmes et de la variabilité de ces

81 Françoise Picq, «Introduction: des mouvements féministes entre spécificité et universalité», dans Ephesia, ouvrage cité, p. 334.

82 Christine Delphy, «Penser le genre: Quels problèmes?», dans Marie Claude Hurtig, Michèle Kail et Hélène Rouch (dir.) ouvrage cité, p. 94.

83 Linda J. Nicholson, «Interpreting gender», Signs, no 20, 1994, p. 79-92.

84 Joan Scott, art. cité. 
différences dans le temps ou l'espace, mais encore pour explorer et revoir leurs divers effets structurants dans la constitution des groupes sociaux. Pour les féministes des différences, la notion de genre représente en quelque sorte l'outil d'analyse permettant de «concevoir le sujet social et les relations de subjectivité à la socialité d'une autre façon: un sujet constitué par le genre et non par la différence sexuelle seule mais plutôt à travers des langages et des représentations culturelles; un sujet "engenré" et engendré dans l'expérimentation de la race et de la classe aussi bien que de la relation sexuelle, un sujet dès lors non pas unifié mais plutôt multiple, et autant divisé que soumis à des contradictions ${ }^{85} \gg$.

La plupart des militantes et intellectuelles féministes adhèrent sans réserve au principe qui appelle au décentrement de l'analyse et de l'action, même si celui-ci trouve beaucoup plus difficilement sa correspondance dans la pratique. Elles jugent utile toutefois de rappeler que l'identité «genrée» ou plutôt «les identités multiples des sujets-femmes ne se construisent pas dans un vacuum ${ }^{86}$ ». En effet, depuis les années quatre-vingt, la plupart des théoriciennes féministes ont cessé de chercher des origines universelles ou uniques au patriarcat; elles ont été unanimes à affirmer le caractère historiquement situé de leur propre regard et de celui des acteurs et actrices; enfin, elles se sont largement préoccupées de développer des analyses plus sensibles à la diversité des expériences vécues par les femmes et à l'incidence des divers facteurs de hiérarchisation. Par contre, si elles sont désormais convaincues qu'il est pertinent de formuler leurs propositions théoriques dans une perspective plurielle et pluraliste, plusieurs se montrent néanmoins réticentes face au réductionnisme socio-politique qui entache certaines des analyses post-structuralistes lorsque des théoriciennes telles Flax, Weedon, Fraser et Nicholson ${ }^{87}$ accolent beaucoup trop rapidement à toutes les écoles de pensée du féminisme l'étiquette d'essentialisme. Formulée à l'emporte-pièce, une telle critique ignore l'idée fondatrice des problématiques féministes radicales matérialistes ou socialistes qui ont effectivement pris forme dans le refus d'expliquer le confinement des femmes dans un état d'infériorité par des arguments d'ordre naturel ou biologique et qui se sont intéressées à la dimension sociale de la division des sexes et la dynamique de sa reproduction dans les différentes sociétés. De même, il y a tout lieu de questionner le point de vue d'auteures qui, telle Weedon, conçoivent le féminisme post-structuraliste comme une option supérieure, sinon comme la première tentative sérieuse de théorisation féministe. Une telle assertion, d'une part, nie l'importante production théorique et empirique qui a fait accéder les études féministes au rang de champ disciplinaire et,

85 Teresa De Lauretis, The Technologies of Gender. Essays on Theory, Film and Fiction, Bloomington, Indiana, University Press, 1987, p. 2 (traduction libre de Micheline De Sève).

86 Micheline De Sève, art. cité, p. 32.

87 Voir Jane Flax, ouvrage cité; Chris Needon, ouvrage cité; et, en particulier, l'introduction de Nancy Fraser et Linda J. Nicholson, «Social criticism without philosophy: An encounter between feminism and postmodernism», dans Linda J. Nicholson (dir.), ouvrage cité, p. 19-38. 
d'autre part, néglige le fait que, depuis plus de trois décennies maintenant, des féministes dissidentes lesbiennes, afro-américaines, du tiers-monde, etc. ont bien fait valoir la pertinence de repenser les femmes non seulement sous l'angle de leur rapport avec la dimension sexuée de leur vie, mais aussi sous l'angle de l'interdépendance de ce rapport avec les autres dimensions de leur expérience sociale et historique. Enfin, le plus inquiétant est, à mes yeux, que l'approche post-moderne ne peut soutenir un projet politique féministe. Et pour cause, puisque ses présupposés mènent au rejet de la dynamique structurante et déterminante des rapports de sexe. Dès lors, l'approche post-moderne contribue à la neutralisation du pouvoir explicatif et subversif des théories féministes et incite à la division plutôt qu'à la mise en commun des savoirs et des expériences des femmes. Bref, parce qu'elle mène au morcellement et à la fragmentation à l'infini des identités et des rapports sociaux, elle constitue un frein à la construction d'un projet concerté, ponctuel ou à long terme, non pas nécessairement consensuel, mais ouvert aux préoccupations de toutes les femmes et de tous les hommes, «sans distinction de couleur, d'orientation sexuelle, d'état civil, de religion, de convictions politiques, de langue, d'origine ethnique, de nationalité, de condition sociale, d'âge et de condition physique et mentale ${ }^{88}$ ».

Cela étant dit, il m'apparaît que l'enjeu actuel, aussi bien théorique que politique, du féminisme à l'aube du prochain millénaire est, selon une observation que j'emprunte à Liliane Kandel ${ }^{89}$, de savoir comment, dans une relation dialectique, «maintenir par les deux bouts la contradiction entre universalisme et particularisme, entre tentations identitaires et projets égalitaires». En quoi consisterait alors une véritable conception féministe de la société? En réalité, peu de féministes, même parmi celles qui s'inspirent de la problématique post-moderne, pensent qu'il serait approprié d'écarter les analyses féministes globales ou les analyses macro-structurelles des inégalités sociales pour forger une problématique plurielle et pluraliste qui place au cœur de son argument la reconnaissance et non l'occultation des différences dans des contextes socio-politiques distincts et mouvants ${ }^{90}$. Pour maintenir un «pôle significatif de transformation sociale [...] dans des sociétés si diverses soient-elles ${ }^{91} »$, plusieurs proposent de formuler, sur la base de l'expérience collective des femmes de la division et de la hiérarchie des rapports de sexe, une critique commune des idéologies et des institutions sociales dominantes et de miser

88 Fédération des femmes du Québec, Marche mondiale des femmes 2000, vol 1, no 1, avril 1998, p. 1.

89 Liliane Kandel, «Féminisme, multiculturalisme, cosmopolitisme: migrations de l'identité dans les mouvements de femmes», dans Ephesia, ouvrage cité, p. 363.

90 Voir l'intéressant article de Sandra Harding, «Feminism, science, and the antienlightenment critiques», dans Linda J. Nicholson (dir.), ouvrage cité, p. 83-106. Voir également, Christine Di Stefano, «Dilemmas of difference: Feminism, modernity, and postmodernism», dans Linda J. Nicholson (dir.), ouvrage cité, p. 63-82.

91 Micheline De Sève, art. cité, p. 25 et 32. 
sur la diversité des expériences des différentes minorités pour mieux déconstruire le sens et la logique de la reconduction des rapports de pouvoir. Dès lors, loin de proposer le maintien d'une fiction homogène du monde des femmes, la sociologie féministe doit se concentrer sur l'élaboration de méthodes et d'outils conceptuels, bien enracinés dans le concret et l'historicité, pour mieux penser la multiplicité et la complexité des processus sociaux sexués et tenter de réconcilier les objectifs de la quête d'égalité des femmes avec leur refus d'assimilation à la norme masculine et avec la reconnaissance d'une identité sociale féminine singulière et multiple.

A l'ambition d'un unanimisme féministe global, à la culture de la différence, se sont donc progressivement substituées une culture féministe des différences et une volonté de mobilisation plurielle, pluraliste et solidaire. On admet en outre, dans l'ensemble, que la pensée féministe doit veiller à ne pas se prêter à la théorisation du désengagement et de l'individualisme, mais surtout doit maintenir une réflexion théorique qui milite en faveur du changement et de la mobilisation pour renverser les rapports de pouvoir, quelles que soient leur forme et leur nature. Pour y arriver, il est proposé non seulement d'entreprendre des actions ponctuelles de coalition ${ }^{92}$, mais également de promouvoir l'adhésion solidaire plutôt que consensuelle du plus grand nombre possible de femmes a un projet féministe dont le rythme, les approches et les expressions seraient diversifiés ${ }^{93}$, tout en maintenant le cap sur l'élimination des processus sociaux sexués de division et de hiérarchisation à l'œuvre dans toutes les sociétés du monde.

Bref, à l'instar de plusieurs théoriciennes et militantes féministes, je me montre réfractaire à l'adoption de positions réductionnistes ou asociales dans lesquelles les énoncés, les pratiques et les projets seraient tous considérés comme équivalents sur la base d'un déconstructionnisme absolu qui n'habilite plus personne à «légitimement parler, étudier ou Prétendre savoir quoi que ce soit en dehors de sa propre expérience ${ }^{94} \gg$ ou encore au nom d'un relativisme culturel sans nuance.

Il me semble en effet que l'avenir des études féministes passe par leur capacité à éviter l'enfermement dans une problématique du singulier, du particulier et des particularismes. Dans cette optique, elles doivent aussi éviter l'effritement illimité et autodestructeur des solidarités, car la survie du féminisme, comme discours et pratiques, dépend de l'existence même d'une base collective de réflexion et d'action relativement unifiée. Par ailleurs, elles devront, l'argument a été invoqué, réussir à mieux conceptualiser la complexité des «identités multiples et simultanées des

\footnotetext{
92 Laura L. Frader, art. cité.

93 C'est notamment l'objectif qui anime les responsables de la Marche de l'an 2000 organisée par la Fédération des femmes du Québec.

94 Susan Moller Okin, art. cité, p. 64.
} 
femmes ${ }^{95}$ », de même que la «sérialité» de leurs positions ${ }^{96}$ dans l'interdépendance des rapports sociaux, tout en cherchant à rallier les femmes autour de thèmes de réflexion communs ou d'actions ponctuelles concertées. Pour conserver leur dynamisme et leur caractère subversif, elles devront donc, en pratique, mieux s'appuyer sur l'expérience concrète et relationnel des femmes pour approfondir leurs connaissances, sur ce qui les rassemble, sans méconnaître ce qui les sépare les unes des autres, et les rapprochent ou les différencient également des hommes, dans un système de rapports qui indéniablement, continue d'être façonné par des cultures et des structures de pouvoir patriarcales. Enfin, sans rejeter pour autant l'idée de s'interroger sur la véritable signification de la féminité et du féminin, les études féministes se doivent d'échapper au romantisme d'une sacralisation d'un féminin-maternel essentiel ou au danger de la «naturalisation» des rapports hommes-femmes de telles tendances étant totalement en contradiction avec la définition des études féministes comprises à la fois comme méthode d'appréhension de la dynamique sociale et pratique socio-politique de changement.

Francine Descarries

Département de sociologie

Université du Québec à Montréal

\section{Résumé}

\section{$\underline{\text { Retour à la table des matières }}$}

En inscrivant les rapports sociaux de sexe au cœur de leur élaboration conceptuelle et la transformation de ces derniers comme enjeu stratégique, les études féministes se sont progressivement imposées dans l'univers des sciences humaines comme démarche critique et problématique du changement. Après avoir rappelé les trois grands courants de pensée qui se sont développés au sein du mouvement des femmes et à sa marge au cours des dernières décennies, l'auteure se demande si la pensée féministe, ou plutôt les pensées féministes actuelles participent encore à ces

95 Elizabeth V. Spelman, Inessential Woman: Problems of Exclusion in Feminist Thought, Boston, Beacon Press, 1988.

96 Iris M. Young, «Gender as seriality: Thinking about women as a social collective», Signs, no 19, 1994, p. 713-738. 
mêmes finalités. Sont-elles toujours en mesure de contribuer au renouvellement des savoirs, de proposer un projet social inédit et d'imaginer les termes d'un nouveau contrat entre les sexes? Sont-elles capables d'alimenter une lutte féministe solidaire, une politique de coalition ouverte aux diversités et aux différences, mais qui ne perdrait pas pour autant sa cohésion et son pouvoir de ralliement? Ces questions servent de fil conducteur à la réflexion de l'auteure.

Mots-clés: rapports sociaux, rapports de sexe, féminisme, études féministes, pensée féministe, mouvement des femmes, différence, identité, contrat social, genre.

Fin du texte 